\title{
MODEL SELECTION FOR REGULARIZED LEAST-SQUARES ALGORITHM IN LEARNING THEORY
}

\author{
E. DE VITO, A. CAPONNETTO, AND L. ROSASCO
}

\begin{abstract}
We investigate the problem of model selection for learning algorithms depending on a continuous parameter. We propose a model selection procedure based on a worst case analysis and data-independent choice of the parameter. For regularized least-squares algorithm we bound the generalization error of the solution by a quantity depending on few known constants and we show that the corresponding model selection procedure reduces to solving a bias-variance problem. Under suitable smoothness condition on the regression function, we estimate the optimal parameter as function of the number of data and we prove that this choice ensures consistency of the algorithm.
\end{abstract}

\section{IntRODUCTION}

A main goal of Learning Theory is the definition of an algorithm that, given a set of examples $\left(x_{i}, y_{i}\right)_{i=1}^{\ell}$, returns a function $f$ such that $f(x)$ is an effective estimate of the output $y$ when a new input $x$ is given. The map $f$ is chosen from a suitable space of functions, called hypothesis space, encoding some a-priori knowledge on the relation between $x$ and $y$.

A learning algorithm is an inference process from a finite set of data based on a model represented by the hypothesis space. If the inference process is correct and the model realistic, as the number of available data increases, we expect the solution to approximate the best possible solution. This property is usually called consistency, see [7], [8], [10], [12] and [21].

A central problem of Learning Theory is a quantitative assessment of the inference property of a learning algorithm. A number of seminal works, see, for instance, [1], [7], [8] and [21], show that the essential feature of an algorithm should be the capability of controlling the complexity of the solution. Roughly speaking, if the model is too complex the algorithm solution overfits the data. In order to overcome overfitting, different complexity measures are introduced, as VC-dimension, see [21], $\mathrm{V}_{\gamma}$-dimension, see [1], and covering numbers, see [7] and [24]. Interestingly, the good behavior of a large class of algorithms has been recently explained also in terms of stability with respect to variations of the given training set, see [4] and [17].

For both approaches it is natural to introduce a parametric family of learning algorithms in which the parameters control the generalization properties. Typical instances of such algorithms are regularized (à la Tikhonov) algorithms, see, for

Date: October 5, 2004.

2000 Mathematics Subject Classification. Primary 68T05, 68P30.

Key words and phrases. Model Selection, Optimal Choice of Parameters, Regularized LeastSquares Algorithm.

Corresponding author: Ernesto De Vito, +390592055193 (phone), +39059370513 (fax). 
instance, [6], [10], [11], [16] and [20]. In this context a central problem is the optimal choice of the parameter as a function of the number of examples.

In this paper we address this issue for the learning algorithms arising in the minimization of the regularized empirical error

$$
\frac{1}{\ell} \sum_{i=1}^{\ell}\left(y_{i}-f\left(x_{i}\right)\right)^{2}+\lambda\|f\|^{2},
$$

where the minimization takes place on a Hilbert space of continuous functions. The corresponding algorithm is usually called regularized least-squares algorithm or regularization networks, see [6], [10], [11] and [16].

In the above functional the first term measures the error of $f$ on the given examples, while the second term is a penalty term that controls the "smoothness" of $f$ in order to avoid overfitting. The parameter $\lambda$ controls the trade-off between these two terms, that is, the balance between the fitting capability of the solution and its complexity.

Our results are in the spirit of [6] and [15]. In particular, our aim is to provide a selection rule for the parameter $\lambda$ which is optimal for any number $\ell$ of examples and provides the desired asymptotic behavior when $\ell$ goes to infinity. As usual, see [7], [10] and [21], we describe the relation between $x$ and $y$ by means of an unknown probability distribution $\rho(x, y)$. Given a solution $f$, the expected risk

$$
\int(y-f(x))^{2} d \rho(x, y)
$$

measures how well the probabilistic relation between $x$ and $y$ is described by the deterministic rule $y=f(x)$. Following [6], the optimal parameter is the one that provides the solution with minimal expectation risk. Since the expected risk is unknown, we need a probabilistic bound on it to have a feasible selection rule. The bound we propose relies on the stability properties of the regularized least-squares algorithm, see [4], and does not depend on any complexity measure on $\mathcal{H}$, which is a central tool in [6] and [15]. A different point of view is given in [20].

The paper is organized as follows. In Section 2 we recall some basic concept of Learning Theory and we discuss the problem of model selection for algorithms depending on a parameter. In Section 3, we specialize this problem to the regularized least-squares algorithm and find a probabilistic bound for the expected risk of the solution, which is the main result of the paper. In Section 4 we estimate the optimal parameter and prove the consistency of the regularized least-squares algorithm.

\section{LEARNing Theory and optimal Choice}

This section is devoted to the following issues. First, we briefly recall the basic concepts of Learning Theory. Second, we discuss the problem of the choice of the parameter for families of learning algorithms labeled by one real valued parameter. In particular, we give some insights into the relation between the Bayesian approach (average case) and the learning approach (worst case), and we propose a general framework for parameter selection in a worst case scenario approach. Finally, we discuss the problem of data dependent choice of the parameter. In the following we assume the reader to have a basic knowledge of Learning Theory (for reviews see [5], [7], [10], [12] and [21]). 
2.1. Learning Theory. In Learning Theory, examples are drawn from two sets: the input space $X$ and the output space $Y$. The relation between the variable $x \in X$ and the variable $y \in Y$ is not deterministic and it is described by a probability distribution $\rho$, which is known only by means of $\ell$ examples $D=\left(\left(x_{1}, y_{1}\right), \ldots,\left(x_{\ell}, y_{\ell}\right)\right)$, drawn identically and independently from $X \times Y$ according to $\rho$. The set of examples $D$ is called training set. For regression problems, as we are dealing in this paper, the output space $Y$ is a subset of real numbers.

The aim of Learning Theory is to learn from a training set a function $f: X \rightarrow Y$, called estimator, such that $f(x)$ is an effective estimate of $y$ when $x$ is given. The inference property of $f$ is measured by its expected risk

$$
I[f]=\int_{X \times Y}(f(x)-y)^{2} d \rho(x, y) .
$$

Since we are dealing with regression problem, the choice of the quadratic loss function is natural. However the discussion of this section holds for a wide class of loss functions (for a discussion of the properties of arbitrary loss function see [10], [18] and $[20])$.

In Learning Theory one approximates the probabilistic relation between $x$ and $y$ by means of functions $y=f(x)$ defined on the input space $X$ and belonging into some a priori given set of model functions, called hypothesis space. For regression problems the model functions $f$ are real valued.

A learning algorithm is a map that, given a training set $D$, outputs an estimator $f_{D}$ chosen in the hypothesis space. A good algorithm is such that the expected risk $I\left[f_{D}\right]$ is as small as possible, at least for generic training sets.

A well known example of learning algorithm is the empirical risk minimization algorithm, see, for instance, [7], [10] and [21]. For a training set $D$, the estimator $f_{D}$ is defined as the one that minimizes the empirical risk

$$
I_{\mathrm{e} m p}^{D}\left[f_{D}\right]=\frac{1}{\ell} \sum_{i=1}^{\ell}\left(f_{D}\left(x_{i}\right)-y_{i}\right)^{2}
$$

over the hypothesis space. Different choices of the hypothesis space give rise to different algorithms, so one usually introduces a sequence of nested hypothesis spaces

$$
\mathcal{H}_{\lambda_{1}} \subset \mathcal{H}_{\lambda_{n}} \subset \ldots \subset \mathcal{H}
$$

where $\lambda_{1}>\lambda_{2}>\ldots \lambda_{n}$ and $\mathcal{H}_{\lambda_{k}}$ is the subset of functions in the model space $\mathcal{H}$ that have complexity less than $\frac{1}{\lambda_{k}}$, according to some suitable measure on complexity (for example the inverse of the norm of $f$, see [7], or its VC-dimension, see [10] and [21]). The regularized least-squares algorithm discussed in the introduction is another example of a learning algorithm: for a training set $D$, the estimator $f_{D}$ is defined as the minimizer of

$$
\frac{1}{\ell} \sum_{i=1}^{\ell}\left(y_{i}-f\left(x_{i}\right)\right)^{2}+\lambda\|f\|^{2},
$$

where the minimum is on a Hilbert space $\mathcal{H}$ of functions defined on $X$ (other loss functions and penalty terms can be considered instead of $\|f\|^{2}$, see [10], [20] and [21] for a discussion).

In both examples there is a family of algorithms labeled by a parameter controlling the complexity of the estimator. The problem of model selection corresponds 
to characterizing a rule for the choice of the parameter in such a way that some criterion of optimality with respect to the inference and consistency properties is satisfied. The following section discusses this question.

2.2. Optimal choice and model selection rule. In the following we consider a family of learning algorithms that are labeled by a positive parameter $\lambda$; we assume that the complexity of the solution decreases with $\lambda$. Given a parameter $\lambda$ and a training set $D$, the algorithm provides an estimator $f_{D}^{\lambda} \in \mathcal{H}$, where $\mathcal{H}$ is some given (vector) space of functions. In this paper the space $\mathcal{H}$ is given, however one can easily extend the present discussion to the case of $\mathcal{H}$ being labeled by some parameters, as kernel parameters when $\mathcal{H}$ is a reproducing kernel Hilbert space, see $[2]$.

In this framework the problem of model selection is the problem of the choice of the optimal parameter $\lambda$. In applications the parameter $\lambda$ is usually chosen through an a posteriori procedure like cross-validation or using a validation set, see, for instance, [22].

To give an a priori definition of the optimal regularization parameter we recall that a good estimator $f_{D}^{\lambda}$ should have small expected risk $I\left[f_{D}^{\lambda}\right]$. So a natural definition for the optimal parameter $\lambda_{o p t}$, is the value of $\lambda$ minimizing $I\left[f_{D}^{\lambda}\right]$. However, this statement needs some careful examination since $I\left[f_{D}^{\lambda}\right]$ is a random variable on the sample space of all training sets. This observation introduces some degree of freedom in the definition of $\lambda_{o p t}$. Following a Bayesian approach, a possible definition is the following one

$$
\lambda_{o p t}:=\underset{\lambda>0}{\operatorname{argmin}} E_{D}\left(I\left[f_{D}^{\lambda}\right]\right),
$$

where $E_{D}$ denotes the expectation with respect to the training sets, see [23]. The above definition can be refined by considering also the variance $\sigma^{2}$ of the random variable, for example considering

$$
\lambda_{o p t}:=\underset{\lambda>0}{\operatorname{argmin}}\left\{E_{D}\left(I\left[f_{D}^{\lambda}\right]\right)+\sigma^{2}\left(I\left[f_{D}^{\lambda}\right]\right)\right\} .
$$

A third possibility is the worst case analysis. Given a confidence level $\eta \in(0,1)$ and defined the quantity

$$
E_{\text {opt }}(\lambda, \eta):=\inf _{t \in[0 ;+\infty)}\left\{t \mid \operatorname{Prob}\left\{D \in Z^{\ell} \mid I\left[f_{D}^{\lambda}\right]>t\right\} \leq \eta\right\}
$$

one lets the optimal parameter be

$$
\lambda_{\text {opt }}(\eta):=\underset{\lambda>0}{\operatorname{argmin}} E_{\text {opt }}(\lambda, \eta) .
$$

We notice that the first two definitions require the knowledge of the first (and second) order momentum of the random variable $I\left[f_{D}^{\lambda}\right]$. This is a satisfactory characterization if we assume to deal with normal distributions. On the other hand it is easy to see that the third definition amounts to a complete knowledge of the random variable $I\left[f_{D}^{\lambda}\right]$. Indeed, given $\lambda, E_{\text {opt }}(\lambda, \eta)$, viewed as function of $1-\eta$, is the inverse of the distribution function of $I\left[f_{D}^{\lambda}\right]$.

However, in Learning Theory, the above definitions have only a theoretical meaning since the distribution $\rho$ and, hence, the random variable $I\left[f_{D}^{\lambda}\right]$ are unknown. To overcome this problem, one studies the random variable $I\left[f_{D}^{\lambda}\right]$ through a known 
probabilistic bound $E(\lambda, \ell, \eta)$ of the form

$$
\operatorname{Prob}\left\{D \in Z^{\ell} \mid I\left[f_{D}^{\lambda}\right]>E(\lambda, \ell, \eta)\right\} \leq \eta .
$$

For the worst case setting the above expression leads to the following model selection rule

$$
\lambda_{0}(\ell, \eta):=\underset{\lambda>0}{\operatorname{argmin}} E(\lambda, \ell, \eta) .
$$

In order to make the above definition rigorous we assume that $E$ extends to a continuous function of $\lambda$ on $[0,+\infty]$ into itself, and replace (5) by

$$
\lambda_{0}(\ell, \eta)=\max \underset{\lambda \in[0,+\infty]}{\operatorname{argmin}} E(\lambda, \ell, \eta) .
$$

The continuity of $E$ ensures that the definition is well stated, even if $\lambda_{0}$ could be occasionally zero or infinite. We select the maximum among the minimizers of $E$ to enforce uniqueness of $\lambda_{0}$ : this choice appears quite natural since it corresponds to the most regular estimator fitting the constraint of minimizing the bound.

Some remarks are in order. First of all, different bounds $E$ give rise to different selection criteria. Moreover to have a meaningful selection rule the bound $E$ has to be a function only of known quantities. In this paper we exhibit a bound that gives rise to an optimal parameter defined through a simple algebraic equation. Second, the random variable $I\left[f_{D}^{\lambda}\right]$ depends on the number $\ell$ of examples in the training set and, as a consequence, the optimal parameter $\lambda_{0}$ is a function of $\ell$. So it is natural to study the asymptotic properties of our selection rule when $\ell$ goes to infinity. In particular, a basic requirement is consistency, that is, the fact that $I\left[f_{D}^{\lambda_{0}(\ell)}\right]$ tends to the smallest attainable expected risk as the number of data goes to infinity. The concept of (weak universal) consistency is formally expressed by the following definition, see [8],

Definition 1. The one parameter family of estimators $f_{D}^{\lambda}$ provided with a model selection rule $\lambda_{0}(\ell)$ is said to be consistent if, for every $\epsilon>0$, it holds

$$
\lim _{\ell \rightarrow \infty} \sup _{\rho} \operatorname{Prob}\left\{D \in Z^{\ell} \mid I\left[f_{D}^{\lambda_{0}(\ell)}\right]>\inf _{f \in \mathcal{H}} I[f]+\epsilon\right\}=0,
$$

where the sup is over the set of all probability measures on $X \times Y$.

In the above definition, the number $\inf _{f \in \mathcal{H}} I[f]$ represents a sort of bias error, [12], associated with the choice of $\mathcal{H}$ and, hence, it can not be controlled by the parameter $\lambda$. In particular if there exists $f_{\mathcal{H}} \in \mathcal{H}$ such that $I\left[f_{\mathcal{H}}\right]=\inf _{f \in \mathcal{H}} I[f]$, the estimator $f_{\mathcal{H}}$ is the best possible deterministic description we can give of the relation between $x$ and $y$, for given $\mathcal{H}$. For sake of clarity, we notice that, for empirical risk minimization algorithm, the bias error is usually called approximation error and it is controlled by the choice of the hypothesis space, see [7] and [15].

2.3. Data dependency. The choices of the parameter $\lambda$ discussed in the above section are a priori since do not depend on the training set $D$. One could also consider a posteriori choices where $\lambda_{0}$ depends on the training set $D$. In a worst case analysis, this corresponds to considering a bound depending explicitly on $D$, that is

$$
\operatorname{Prob}\left\{D \in Z^{\ell} \mid I\left[f_{D}^{\lambda}\right]>E(\lambda, \ell, \eta, D)\right\} \leq \eta
$$


A well-known example of the above bound is the principle of structural risk minimization, see [10] and [21]. For Empirical Risk Minimization algorithm in nested hypothesis spaces

$$
\mathcal{H}_{\lambda_{1}} \subset \mathcal{H}_{\lambda_{n}} \subset \ldots \subset \mathcal{H}
$$

the parameter $\lambda$ is chosen in order to minimize the bound

$$
E(\lambda, \ell, \eta, D)=I_{\mathrm{e} m p}^{D}\left[f_{D}^{\lambda}\right]+\mathcal{G}(\lambda, \ell, \eta, D),
$$

where $\mathcal{G}(\lambda, \ell, \eta, D)$ is a term that controls the complexity of the solution. Usually, $\mathcal{G}$ does not dependent on the training set since the measure of complexity is uniform on the hypothesis space $\mathcal{H}_{\lambda}$. However, we get a dependence of the bound on $D$ because of the presence of the empirical term $I_{\mathrm{e} m p}^{D}\left[f_{D}^{\lambda}\right]$ in Eq. (8).

Now, mimicking the idea of previous discussion, we could define the optimal parameter as

$$
\lambda_{0}(\ell, \eta, D):=\underset{\lambda>0}{\operatorname{argmin}} E(\lambda, \ell, \eta, D) .
$$

Clearly, we get a dependence of the optimal parameter on the training set $D$. This dependence can be, in principle, problematic due to the probabilistic nature of Eq. (4). Indeed, for every $\lambda$, we can define the collection of good training sets for which the bound is tight, i.e.

$$
\mathcal{A}(\lambda)=\left\{D \in Z^{\ell} \mid I\left[f_{D}^{\lambda}\right] \leq E(\lambda, \ell, \eta, D)\right\} .
$$

By definition of the bound $E$, the probability of drawing a good training set $D \in$ $\mathcal{A}(\lambda)$ is greater than $1-\eta$. However, the previous confidence level cannot be applied to $I\left[f_{D}^{\lambda_{0}(D)}\right]$. Indeed, it can happen that the probability of drawing a training set $D$ in the set

$$
\left\{D \in Z^{\ell} \mid I\left[f_{D}^{\lambda_{0}(D)}\right] \leq E\left(\lambda_{0}(D), \ell, \eta, D\right)\right\}=\left\{D \in Z^{\ell} \mid D \in \mathcal{A}\left(\lambda_{0}(D)\right)\right\}
$$

could be much smaller that $1-\eta$, depending on the structure of the sets $\mathcal{A}(\lambda)$ in the sample space $Z^{\ell}$. Simple toy examples of this pathology can be built.

A possible solution to this kind of problem requires further analyses, see, for instance, [3], [8] and [21]. In this paper we avoid the problem by considering data independent bounds and hence a priori model selection rules.

\section{A PROBABILISTIC BOUND FOR REGULARIZED LEAST-SQUARES ALGORITHM}

We consider throughout the problem of model selection for regularized leastsquares algorithm in the regression setting.

In the present section we first show that the expected risk of the estimator $f_{D}^{\lambda}$ concentrates around the expected risk of $f^{\lambda}$, where $f^{\lambda}$ is the minimizer of the regularized expected risk $I[f]+\lambda\|f\|_{\mathcal{H}}^{2}$.

Moreover, we give a probabilistic bound of the difference between $I\left[f_{D}^{\lambda}\right]$ and $I\left[f^{\lambda}\right]$ in terms of a function $S(\lambda, \ell, \eta)$ depending on the parameter $\lambda$, the number of examples $\ell$ and the confidence level $1-\eta$. Our results are based on stability properties of regularized least-squares algorithm, see [4], and the McDiarmid concentration inequality, see [13]. In particular, we do not make use of any complexity measure on the hypothesis space, like VC-dimension, see [21], or covering number, see [7] and [15]. We stress that the bound $S$ depends on $\mathcal{H}$ only through two simple constants related to topological properties of $X$ and $Y$.

Compared to previous results (see, for instance, [3], [4], [7], [14], [15], [21]) we are not interested into the deviation of the empirical risk from the expected risk 
and we bound directly the expected risk of the estimator $f_{D}^{\lambda}$. Moreover, our result concentrates $I\left[f_{D}^{\lambda}\right]$ around $I\left[f^{\lambda}\right]$ both from above and below so that $I\left[f^{\lambda}\right]$ will play the role of approximation error and $S(\lambda, \ell, \eta)$ the role of sample error (our terminology is close to the definition of [6], which is somewhat different with the notation of [7] and [15]).

Finally, in order to obtain algorithmically computable results, we make some smoothness assumption on the probability distribution $\rho$. By means of standard results in approximation theory, see [19], we find a bound $E$, depending only on known quantities.

Before stating the main theorem of the section we set the notations.

3.1. Notations. We assume that the input space $X$ is a compact subset of $\mathbb{R}^{d}$ and the output space $Y$ is a compact subset of $\mathbb{R}$. The assumption of compactness is for technical reasons and simplifies some proof.

We let $\rho$ be the unknown probability measure on $Z=X \times Y$ describing the relation between $x \in X$ and $y \in Y$, and $\nu$ the marginal distribution of $\rho$ on $X$. Moreover, for $\nu$-almost all $x \in X$, let $\rho_{x}$ be the conditional distribution of $y$ with respect to $x$.

If $\xi$ is a random variable on $Z$, we denote its mean value by $E_{Z}(\xi)$,

$$
E_{Z}(\xi)=\int_{X \times Y} \xi(x, y) d \rho(x, y) .
$$

As usual, $L^{2}(X, \nu)$ is the Hilbert space of square-integrable functions and $\|\cdot\|_{\nu}$, $\langle\cdot, \cdot\rangle_{\nu}$ are the corresponding norm and scalar product.

We let $\mathcal{C}(X)$ be the space of (real) continuous functions on $X$ equipped with the uniform norm, $\|f\|_{\infty}=\sup _{x \in X}|f(x)|$.

We denote by $f_{0}$ and $\sigma_{0}$ the regression and noise functions defined respectively as

$$
\begin{aligned}
f_{0}(x) & =\int_{Y} y d \rho_{x}(y), \\
\sigma_{0}^{2}(x) & =\left(\int_{Y} y^{2} d \rho_{x}(y)\right)-\left(f_{0}(x)\right)^{2},
\end{aligned}
$$

that belong to $L^{2}(X, \nu)$ due to the compactness of $X$ and $Y$.

Given $\ell \in \mathbb{N}$, let $Z^{\ell}$ be the set of all training sets with $\ell$ examples. We regard $Z^{\ell}$ as a probability space with respect to the product measure $\rho^{\ell}=\rho \otimes \ldots \otimes \rho$. If $\xi$ is a random variable on $Z^{\ell}$ we denote its mean value by $E_{D}(\xi)$,

$$
E_{D}(\xi)=\int_{Z^{\ell}} \xi(D) d \rho^{\ell}(D)
$$

Given $D \in Z^{\ell}$, let $\rho_{D}$ be the empirical measure on $X \times Y$ defined by $D$, that is

$$
\rho_{D}=\frac{1}{\ell} \sum_{i=1}^{\ell} \delta_{x_{i}} \delta_{y_{i}}
$$

where $\delta_{x}$ and $\delta_{y}$ are the Dirac measures at $x$ and $y$ respectively.

We assume the hypothesis space $\mathcal{H}$ be a reproducing kernel Hilbert space with a continuous kernel $K: X \times X \rightarrow \mathbb{R}$. The assumption on the kernel ensures $\mathcal{H}$ being a 
Hilbert space of continuous functions. We let $\|\cdot\|_{\mathcal{H}}$ and $\langle\cdot, \cdot\rangle_{\mathcal{H}}$ be the corresponding norm and scalar product. We define

$$
\begin{aligned}
K_{x}(s) & =K(s, x) \quad x \in X \\
\kappa & =\sup \{\sqrt{K(x, x)} \mid x \in X\} \\
\delta & =\sup \{|y| \mid y \in Y\} .
\end{aligned}
$$

It is well known, see [2] and [7], that

$$
\begin{aligned}
\mathcal{H} & \subset \mathcal{C}(X) \subset L^{2}(X, \nu), \\
f(x) & =\left\langle f, K_{x}\right\rangle_{\mathcal{H}}, \quad x \in X \\
\|\cdot\|_{\nu} & \leq\|\cdot\|_{\infty} \leq \kappa\|\cdot\|_{\mathcal{H}} .
\end{aligned}
$$

We denote by $L_{\nu}$ the integral operator on $L^{2}(X, \nu)$ with kernel $K$, that is,

$$
\left(L_{\nu} f\right)(s)=\int_{X} K(s, x) f(x) d \nu(x) \quad s \in X
$$

and by $P$ the projection onto the closure of the range of $L_{\nu}$. In particular, one has that the closure of $\mathcal{H}$ with respect the norm of $L^{2}(X, \nu)$ is $P L^{2}(X, \nu)$.

We recall that, given $f \in L^{2}(X, \nu)$, the expected risk of $f$ is

$$
I[f]=\int_{X \times Y}(y-f(x))^{2} d \rho(x, y) .
$$

We let $I_{\mathcal{H}}$ be the bias error

$$
I_{\mathcal{H}}=\inf _{f \in \mathcal{H}} I[f]
$$

For any $\lambda>0$ we denote by $f^{\lambda}$ the solution of

$$
\min _{f \in \mathcal{H}}\left\{I[f]+\lambda\|f\|_{\mathcal{H}}^{2}\right\}
$$

which exists and it is unique, see, for instance, [6].

Finally, given $D \in Z^{\ell}$, the empirical risk of $f \in \mathcal{C}(X)$ is given by

$$
I_{\mathrm{e} m p}^{D}[f]=\frac{1}{\ell} \sum_{i=1}^{\ell}\left(y_{i}-f\left(x_{i}\right)\right)^{2} .
$$

For all $\lambda>0$, the estimator $f_{D}^{\lambda}$ is defined as the unique solution of

$$
\min _{f \in \mathcal{H}}\left\{I_{\mathrm{e} m p}^{D}[f]+\lambda\|f\|_{\mathcal{H}}^{2}\right\}
$$

which exists and it is unique, see [6].

In the following we will always consider the square root of the unbiased risk that we indicate with $\mathcal{R}[f]$ to simplify the notation, that is

$$
\mathcal{R}[f]=\sqrt{I[f]-I_{\mathcal{H}}} .
$$

Indeed, Eq. (21) below will show that this quantity can conveniently be interpreted as a distance in $L^{2}(X, \nu)$. 
3.2. Main results. The following theorem shows that, given the parameter $\lambda$, the expected risk of the estimator $f_{D}^{\lambda}$ provided by the regularized least-squares algorithm concentrates around the value $I\left[f^{\lambda}\right]$. Moreover, the deviation can be bounded by a simple function $S$ depending only on the confidence level, the number of examples and two constants, $\kappa$ and $\delta$, encoding some topological properties of $X, Y$ and the kernel (see Eqs. (11) and (12)).

Theorem 1. Given $0<\eta<1, \ell \in \mathbb{N}$ and $\lambda>0$, with probability at least $1-\eta$,

$$
\left|\mathcal{R}\left[f_{D}^{\lambda}\right]-\mathcal{R}\left[f^{\lambda}\right]\right| \leq S(\lambda, \ell, \eta)
$$

where

$$
S(\lambda, \ell, \eta)=\frac{\delta \kappa^{2}}{\lambda \sqrt{\ell}}\left(1+\frac{\kappa}{\sqrt{\lambda}}\right)\left(1+\sqrt{2 \log \frac{2}{\eta}}\right) .
$$

The proof of the theorem is postponed to Section 3.3 after a brief discussion and some remarks on the result.

Let us interpret the quantities occurring in our inequality. The data independent term $\mathcal{R}\left[f^{\lambda}\right]$ can be interpreted as the price payed by replacing the regression function $f_{0}$ with the regularized solution $f^{\lambda}$, in short as the approximation error, compare with [6] and [15].

The term $S(\lambda, \ell, \eta)$ is a bound on $\left|\mathcal{R}\left[f_{D}^{\lambda}\right]-\mathcal{R}\left[f^{\lambda}\right]\right|$, that is on the sample error made by approximating $f^{\lambda}$ through a finite training set $D$, compare with [6], [15] and $[21]$.

Since Theorem 1 bounds $\mathcal{R}\left[f_{D}^{\lambda}\right]$ both from above and below and $S(\lambda, \ell, \eta)$ goes to zero for $\ell$ going to $+\infty$, the expected risk of $f_{D}^{\lambda}$ concentrates around the expected risk of $f^{\lambda}$. Then the splitting of $\mathcal{R}\left[f_{D}^{\lambda}\right]$ into approximation error and sample error appears quite natural and intrinsic to the problem.

3.3. Proofs. In the following, before dealing with the main result, we briefly sketch the scheme of the proof and we show some useful lemmas.

The proof of Theorem 1 is essentially based on two steps

- we show that regularized least-squares algorithm satisfies a kind of stability property with respect to variation of the training set, compare with [4];

- we give an estimate of the mean value of $\mathcal{R}\left[f_{D}^{\lambda}\right]$ (and hence of the mean value of the expected risk).

More precisely, given $\lambda$, we regard $\mathcal{R}\left[f_{D}^{\lambda}\right]$ as a real random variable on $Z^{\ell}$ and we apply the McDiarmid inequality, see [13]. This inequality tells us that

$$
\operatorname{Prob}\left\{D \in Z^{\ell}|| \mathcal{R}\left[f_{D}^{\lambda}\right]-E_{D}\left(\mathcal{R}\left[f_{D}^{\lambda}\right]\right) \mid \geq \epsilon\right\} \leq 2 e^{-\frac{2 \epsilon^{2}}{\sum_{i=1}^{\ell} c_{i}^{2}}}
$$

provided that

$$
\sup _{D \in Z^{\ell}} \sup _{\left(x^{\prime}, y^{\prime}\right) \in Z}\left|\mathcal{R}\left[f_{D}^{\lambda}\right]-\mathcal{R}\left[f_{D^{i}}^{\lambda}\right]\right| \leq c_{i}
$$

where $D^{i}$ is the training set with the $i^{\text {th }}$ example being replaced by $\left(x^{\prime}, y^{\prime}\right)$. 
To work out the proof, we recall some preliminary facts. Since we are considering the quadratic loss the expected risk of $f \in \mathcal{H}$ can be written in the following way,

$$
\begin{aligned}
I[f] & =\left\|f-f_{0}\right\|_{\nu}^{2}+\left\|\sigma_{0}\right\|_{\nu}^{2} \\
& =\left\|P\left(f-f_{0}\right)\right\|_{\nu}^{2}+\left\|(I-P)\left(f-f_{0}\right)\right\|_{\nu}^{2}+\left\|\sigma_{0}\right\|_{\nu}^{2} \\
& =\left\|f-P f_{0}\right\|_{\nu}^{2}+\left\|(I-P)\left(f-f_{0}\right)\right\|_{\nu}^{2}+\left\|\sigma_{0}\right\|_{\nu}^{2} \\
& =\left\|f-P f_{0}\right\|_{\nu}^{2}+\left\|(I-P) f_{0}\right\|_{\nu}^{2}+\left\|\sigma_{0}\right\|_{\nu}^{2}
\end{aligned}
$$

where $f_{0}$ and $\sigma_{0}$ are given by Eqs. (9) and (10), and $(I-P) f=0$ since $f \in \mathcal{H} \subset$ $P L^{2}(X, \nu)$.

It follows that $I_{\mathcal{H}}=\inf _{f \in \mathcal{H}} I[f]=\left\|(I-P) f_{0}\right\|_{\nu}^{2}+\left\|\sigma_{0}\right\|_{\nu}^{2}$ and

$$
\mathcal{R}[f]=\sqrt{I[f]-I_{\mathcal{H}}}=\left\|f-P f_{0}\right\|_{\nu} .
$$

We now recall the explicit form of the minimizers of (15) and (16). One has that

$$
\begin{aligned}
& f^{\lambda}=(T+\lambda)^{-1} g_{\rho} \\
& f_{D}^{\lambda}=\left(T_{\mathbf{x}}+\lambda\right)^{-1} g_{D}
\end{aligned}
$$

where $T$ and $T_{\mathbf{x}}$ are positive operators on $\mathcal{H}$ given by

$$
\begin{aligned}
T & =\int_{X}\left\langle\cdot, K_{x}\right\rangle_{\mathcal{H}} K_{x} d \nu(x) \\
T_{\mathbf{x}} & =\frac{1}{\ell} \sum_{i=1}^{\ell}\left\langle\cdot, K_{x_{i}}\right\rangle_{\mathcal{H}} K_{x_{i}}
\end{aligned}
$$

and $g_{\rho}$ and $g_{D}$ are functions in $\mathcal{H}$ defined by

$$
\begin{aligned}
g_{\rho} & =\int_{X} K_{x} f_{0}(x) d \nu(x) \\
g_{D} & =\frac{1}{\ell} \sum_{i=1}^{\ell} y_{i} K_{x_{i}},
\end{aligned}
$$

(in Eq. (24) the integral is taken with respect to the trace operator norm, while in Eq. (26) the integral is with respect to the norm of $\mathcal{H}$. Both integrals are finite since the integrands are continuous and $X$ is compact).

In order to present the proof of Theorem 1 we need some preliminary Lemmas. The first one provides an upper bound on $\left\|f_{D}^{\lambda}\right\|_{\mathcal{H}}$. The proof is standard and we report it for completeness, see, for instance, [4].

Lemma 1. For all $\lambda>0$,

$$
\left\|f_{D}^{\lambda}\right\|_{\mathcal{H}} \leq \frac{\delta}{\sqrt{\lambda}}
$$

Proof. Since by definition, see Eq. (16),

$$
f_{D}^{\lambda}=\underset{f \in \mathcal{H}}{\operatorname{argmin}}\left\{I_{\mathrm{e} m p}^{D}[f]+\lambda\|f\|_{\mathcal{H}}^{2}\right\},
$$

with the choice $f=0$, it follows that

$$
I_{\mathrm{e} m p}^{D}\left[f_{D}^{\lambda}\right]+\lambda\left\|f_{D}^{\lambda}\right\|_{\mathcal{H}}^{2} \leq I_{\mathrm{e} m p}^{D}[0]+\lambda\|0\|_{\mathcal{H}}^{2}=\frac{1}{\ell} \sum_{i=1}^{\ell} y_{i}^{2} \leq \delta^{2},
$$


where in the last inequality we recalled the definition of $\delta$, see Eq. (12). The thesis follows from the fact that $I_{\mathrm{e} m p}^{D}\left[f_{D}^{\lambda}\right] \geq 0$.

The next step is the estimate of the expectation value of $\left\|T_{\mathbf{x}}-T\right\|$ (here $\|\cdot\|$ denotes the operator norm in $\mathcal{H}$ ) and that of $\left\|g_{D}-g_{\rho}\right\|_{\mathcal{H}}$. To this aim, we recall the following property regarding vector valued random variables on $Z$. Let $\mathcal{K}$ be a Hilbert space and $\xi$ a $\mathcal{K}$-valued random variable on $Z$, then

$$
\begin{aligned}
E_{D}\left(\left\|\frac{1}{\ell} \sum_{i=1}^{\ell} \xi\left(x_{i}, y_{i}\right)-E_{Z}(\xi)\right\|_{\mathcal{K}}\right)^{2} & \leq E_{D}\left(\left\|\frac{1}{\ell} \sum_{i=1}^{\ell} \xi\left(x_{i}, y_{i}\right)-E_{Z}(\xi)\right\|_{\mathcal{K}}^{2}\right) \\
& =\frac{1}{\ell}\left(E_{Z}\left(\|\xi\|_{\mathcal{K}}^{2}\right)-\left\|E_{Z}(\xi)\right\|_{\mathcal{K}}^{2}\right) .
\end{aligned}
$$

The first inequality is consequence of Schwarz inequality and the equality is a straightforward extension of the well-known property of real random variables, see [9].

Lemma 2. Let $a_{1}=\int_{X \times X}\left(K(x, x)^{2}-K\left(x, x^{\prime}\right)^{2}\right) d \nu(x) d \nu\left(x^{\prime}\right)$, then

$$
\begin{aligned}
E_{D}\left(\left\|T_{\mathbf{x}}-T\right\|\right) & \leq \sqrt{\frac{a_{1}}{\ell}} \\
& \leq \frac{\kappa^{2}}{\sqrt{\ell}} .
\end{aligned}
$$

Proof. Let $B_{2}(\mathcal{H})$ be the Hilbert space of selfadjoint Hilbert-Schmidt operators on $\mathcal{H}$ with scalar product $\langle A, B\rangle_{B_{2}(\mathcal{H})}=\operatorname{Tr}(A B)$. Notice that, for all $x \in X$, the rank one operator $\left\langle\cdot, K_{x}\right\rangle_{\mathcal{H}} K_{x}$ is in $B_{2}(\mathcal{H})$, so we can define the $B_{2}(\mathcal{H})$-valued random variable $\xi$ as

Since $\operatorname{Tr}\left(\left\langle\cdot, K_{x}\right\rangle_{\mathcal{H}} K_{x^{\prime}}\right)=\left\langle K_{x^{\prime}}, K_{x}\right\rangle_{\mathcal{H}}$ then

$$
\xi(x)=\left\langle\cdot, K_{x}\right\rangle_{\mathcal{H}} K_{x} .
$$

$$
\begin{aligned}
\left\|\xi(x)-\xi\left(x^{\prime}\right)\right\|_{B_{2}(\mathcal{H})}^{2} & =\operatorname{Tr}\left(\left(\xi(x)-\xi\left(x^{\prime}\right)\right)^{2}\right) \\
& =K^{2}(x, x)+K^{2}\left(x^{\prime}, x^{\prime}\right)-2 K^{2}\left(x^{\prime}, x\right),
\end{aligned}
$$

which implies the continuity of $\xi$. Its mean value is given by

$$
\begin{aligned}
E_{Z}(\xi) & =\int_{X}\left\langle\cdot, K_{x}\right\rangle_{\mathcal{H}} K_{x} d \nu(x) \\
& =T .
\end{aligned}
$$

Now, observe that

$$
\begin{aligned}
E_{D}\left(\|\xi\|_{B_{2}(\mathcal{H})}^{2}\right) & =E_{D}\left(\operatorname{Tr}\left(\left\langle\cdot, K_{x}\right\rangle_{\mathcal{H}} K_{x} K(x, x)\right)\right) \\
& =\int_{X} K(x, x)^{2} d \nu(x)
\end{aligned}
$$

Finally,

$$
\begin{aligned}
\left\|E_{Z}(\xi)\right\|_{B_{2}(\mathcal{H})}^{2} & =\operatorname{Tr}\left(T^{2}\right) \\
& =\int_{X \times X} \operatorname{Tr}\left(\left\langle\cdot, K_{x}\right\rangle_{\mathcal{H}} K_{x^{\prime}} K\left(x, x^{\prime}\right)\right) d \nu(x) d \nu\left(x^{\prime}\right) \\
& =\int_{X \times X} K\left(x, x^{\prime}\right)^{2} d \nu(x) d \nu\left(x^{\prime}\right) .
\end{aligned}
$$


Applying Eq. (28) and the definition of $a_{1}$, one has that

$$
E_{D}\left(\left\|T_{\mathbf{x}}-T\right\|_{B_{2}(\mathcal{H})}\right) \leq \sqrt{\frac{a_{1}}{\ell}}
$$

The thesis follows observing that

$$
\left\|T_{\mathbf{x}}-T\right\| \leq\left\|T_{\mathbf{x}}-T\right\|_{B_{2}(\mathcal{H})}
$$

and

$$
a_{1} \leq \int_{X} K(x, x)^{2} d \nu(x) \leq \kappa^{4}
$$

Lemma 3. Let $a_{2}=\int_{Z} y^{2} K(x, x) d \rho(x, y)-\left\|g_{\rho}\right\|_{\mathcal{H}}^{2}$, then

$$
\begin{aligned}
E_{D}\left(\left\|g_{D}-g_{\rho}\right\|_{\mathcal{H}}\right) & \leq \sqrt{\frac{a_{2}}{\ell}} \\
& \leq \frac{\delta \kappa}{\sqrt{\ell}} .
\end{aligned}
$$

Proof. We follow the scheme of the previous proof. Let $\xi$ be the $\mathcal{H}$-valued random variable

$$
\xi(x, y)=y K_{x}
$$

Since

$$
\left\|\xi(x, y)-\xi\left(x^{\prime}, y^{\prime}\right)\right\|_{\mathcal{H}}^{2}=y^{2} K(x, x)+y^{\prime 2} K\left(x^{\prime}, x^{\prime}\right)-2 y y^{\prime} K\left(x, x^{\prime}\right)
$$

$\xi$ is continuous and

$$
\begin{aligned}
E_{Z}(\xi) & =\int_{X \times Y} y K_{x} d \rho(x, y) \\
& =g_{\rho},
\end{aligned}
$$

by definition of $g_{\rho}$.

Moreover

$$
E_{Z}\left(\|\xi\|_{\mathcal{H}}^{2}\right)=\int_{X \times Y} y^{2} K(x, x) d \rho(x, y)
$$

Applying Eq. (28) and the definition of $a_{2}$, one has that

$$
E_{D}\left(\left\|g_{D}-g_{\rho}\right\|_{\mathcal{H}}\right) \leq \frac{a_{2}}{\ell}
$$

The thesis follows observing that

$$
0 \leq a_{2} \leq \int_{X \times Y} y^{2} K(x, x) d \rho(x, y) \leq \delta^{2} \kappa^{2} .
$$

The next lemma estimates the expectation value of $\mathcal{R}\left[f_{D}^{\lambda}\right]$.

Lemma 4. Following the above notations

$$
\left|E_{D}\left(\mathcal{R}\left[f_{D}^{\lambda}\right]\right)-\mathcal{R}\left[f^{\lambda}\right]\right| \leq \frac{\kappa^{2} \delta}{\lambda \sqrt{\ell}}\left(1+\frac{\kappa}{\sqrt{\lambda}}\right)
$$


Proof. By means of Eq. (23),

$$
\begin{aligned}
f_{D}^{\lambda} & =\left(T_{\mathbf{x}}+\lambda\right)^{-1} g_{D} \\
& =(T+\lambda)^{-1}\left(g_{D}-g_{\rho}\right) \\
& +(T+\lambda)^{-1}\left(T-T_{\mathbf{x}}\right)\left(T_{\mathbf{x}}+\lambda\right)^{-1} g_{D} \\
& +(T+\lambda)^{-1} g_{\rho},
\end{aligned}
$$

that is, using Eq. (22),

$$
f_{D}^{\lambda}-f^{\lambda}=(T+\lambda)^{-1}\left(g_{D}-g_{\rho}\right)+(T+\lambda)^{-1}\left(T-T_{\mathbf{x}}\right) f_{D}^{\lambda} .
$$

Using Eq. (21) and the triangular inequality we have that

$$
\begin{aligned}
\left|\mathcal{R}\left[f_{D}^{\lambda}\right]-\mathcal{R}\left[f^{\lambda}\right]\right| & \leq\left\|f_{D}^{\lambda}-f^{\lambda}\right\|_{\nu} \\
(\text { Eq. }(13)) & \leq \kappa\left\|(T+\lambda)^{-1}\left(g_{D}-g_{\rho}\right)\right\|_{\mathcal{H}} \\
& +\kappa\left\|(T+\lambda)^{-1}\left(T-T_{\mathbf{x}}\right) f_{D}^{\lambda}\right\|_{\mathcal{H}} \\
& \leq \frac{\kappa}{\lambda}\left\|g_{D}-g_{\rho}\right\|_{\mathcal{H}}+\frac{\kappa \delta}{\lambda^{\frac{3}{2}}}\left\|T-T_{\mathbf{x}}\right\|,
\end{aligned}
$$

where $\left\|(T+\lambda)^{-1}\right\| \leq \frac{1}{\lambda}$ and we used Lemma 1 to bound $\left\|f_{D}^{\lambda}\right\|_{\mathcal{H}}$.

Finally we take the mean value on $D$ and use Lemmas 2 and 3 .

We are now ready to prove the main result of the Section.

Proof of Theorem 1. The proof uses the McDiarmid inequality (18). Due to Eq. (21), the conditions (19) become

$$
\sup _{D \in Z^{\ell}} \sup _{\left(x^{\prime}, y^{\prime}\right) \in Z}\left|\left\|f_{D}^{\lambda}-P f_{0}\right\|_{\nu}-\left\|f_{D^{i}}^{\lambda}-P f_{0}\right\|_{\nu}\right| \leq c_{i}
$$

(we recall that $D^{i}$ is the training set where the $i^{\text {th }}$ example is replaced by $\left(x^{\prime}, y^{\prime}\right)$ ).

In order to compute the constants $c_{i}$, we notice that $f_{D}^{\lambda}$ can be decomposed, by means of Eq. (23), as

$$
\begin{aligned}
f_{D}^{\lambda} & =\left(T_{\mathbf{x}}+\lambda\right)^{-1} g_{D} \\
& =\left(T_{\mathbf{x}}+\lambda\right)^{-1}\left(g_{D}-g_{D^{i}}\right) \\
& +\left(T_{\mathbf{x}}+\lambda\right)^{-1}\left(T_{\mathbf{x}^{i}}-T_{\mathbf{x}}\right)\left(T_{\mathbf{x}^{i}}+\lambda\right)^{-1} g_{D^{i}} \\
& +\left(T_{\mathbf{x}^{i}}+\lambda\right)^{-1} g_{D^{i}},
\end{aligned}
$$

that is, using again Eq. (23) with $D^{i}$,

$$
f_{D}^{\lambda}-f_{D^{i}}^{\lambda}=\left(T_{\mathbf{x}}+\lambda\right)^{-1}\left(g_{D}-g_{D^{i}}\right)+\left(T_{\mathbf{x}}+\lambda\right)^{-1}\left(T_{\mathbf{x}^{i}}-T_{\mathbf{x}}\right) f_{D^{i}}^{\lambda} .
$$

By the triangular inequality, we can write

$$
\begin{aligned}
\left|\left\|f_{D}^{\lambda}-P f_{0}\right\|_{\nu}-\left\|f_{D^{i}}^{\lambda}-P f_{0}\right\|_{\nu}\right| & \leq\left\|f_{D}^{\lambda}-f_{D^{i}}^{\lambda}\right\|_{\nu} \\
(\text { Eq. (13) }) & \leq \kappa\left\|\left(T_{\mathbf{x}}+\lambda\right)^{-1}\left(g_{D}-g_{D^{i}}\right)\right\|_{\mathcal{H}} \\
& +\kappa\left\|\left(T_{\mathbf{x}}+\lambda\right)^{-1}\left(T_{\mathbf{x}}-T_{\mathbf{x}^{i}}\right) f_{D^{i}}^{\lambda}\right\|_{\mathcal{H}} \\
& \leq \frac{2 \delta \kappa^{2}}{\lambda \ell}\left(1+\frac{\kappa}{\sqrt{\lambda}}\right) \\
& =: c_{i}
\end{aligned}
$$


where we used Lemma 1 to bound $\left\|f_{D^{i}}^{\lambda}\right\|_{\mathcal{H}}$ and

$$
\begin{aligned}
\left\|\left(T_{\mathbf{x}}+\lambda\right)^{-1}\right\| & \leq \frac{1}{\lambda} \\
\left\|T_{\mathbf{x}}-T_{\mathbf{x}^{i}}\right\| & =\frac{1}{\ell}\left\|\left\langle\cdot, K_{x_{i}}\right\rangle_{\mathcal{H}} K_{x_{i}}-\left\langle\cdot, K_{x_{i}^{\prime}}\right\rangle_{\mathcal{H}} K_{x_{i}^{\prime}}\right\| \\
& \leq \frac{1}{\ell} 2 \kappa^{2}, \\
\left\|g_{D}-g_{D^{i}}\right\|_{\mathcal{H}} & =\frac{1}{\ell}\left\|y K_{x_{i}}-y_{i}^{\prime} K_{x_{i}^{\prime}}\right\|_{\mathcal{H}} \\
& \leq \frac{1}{\ell} 2 \delta \kappa .
\end{aligned}
$$

Plugging the constants $c_{i}$ into McDiarmid inequality (18), we have that

$$
\left|\mathcal{R}\left[f_{D}^{\lambda}\right]-E_{D}\left(\mathcal{R}\left[f_{D}^{\lambda}\right]\right)\right| \leq \epsilon
$$

with probability

$$
1-2 e^{-\frac{\epsilon^{2}}{2\left(\frac{\delta \kappa^{2}}{\lambda \sqrt{\ell}}\left(1+\frac{\kappa}{\sqrt{\lambda}}\right)\right)^{2}}}=1-\eta
$$

so, with probability at least $1-\eta$,

$$
\left|\mathcal{R}\left[f_{D}^{\lambda}\right]-E_{D}\left(\mathcal{R}\left[f_{D}^{\lambda}\right]\right)\right| \leq \frac{\delta \kappa^{2}}{\lambda \sqrt{\ell}}\left(1+\frac{\kappa}{\sqrt{\lambda}}\right) \sqrt{2 \log \frac{2}{\eta}}
$$

The above bound together with Lemma 4, once again by the triangular inequality, completes the proof.

\section{Estimate of the optimal PARAmeter}

We are now in the position to apply the results of the previous Section to the actual estimate of the regularization parameter, following the technique presented in Section 2.

From Theorem 1 we can easily derive the following bound

$$
\mathcal{R}\left[f_{D}^{\lambda}\right] \leq \mathcal{R}\left[f^{\lambda}\right]+S(\lambda, \ell, \eta)
$$

which holds with probability at least $1-\eta$.

From the explicit form of $S(\lambda, \ell, \eta)$, we have that $S(\lambda, \ell, \eta)$ decreases with $\lambda$ and goes to $+\infty$ when $\lambda$ goes to 0 . On the other hand, it is easy to check that $\mathcal{R}\left[f^{\lambda}\right]$ is an increasing function of $\lambda$ and goes to 0 for $\lambda$ going to 0 , see [6].

The bound (29) is of the form of Eq. (4) and can be used in the model selection rule defined by Eq. (6). Our definition ensures existence and uniqueness of the estimate $\lambda_{0}$ of the optimal parameter, however we still have to prove that $\lambda_{0}$ is finite. We now prove that the bound in Eq. (29) provides an estimate $\lambda_{0}$ that is finite for large enough $\ell$.

We consider a slightly more general case that will be useful in the following. We let $A(\lambda)$ be an upper bound on the approximation error, that is, an increasing, continuous function from $[0,+\infty]$ to $[0,+\infty]$ satisfying

$$
A(\lambda) \geq \mathcal{R}\left[f^{\lambda}\right]
$$

and

$$
\lim _{\lambda \rightarrow 0} A(\lambda)=0
$$


The following proposition highlights the special role played by the approximation error $\mathcal{R}\left[f^{\lambda}\right]$ with respect to an arbitrary data independent bound of the form given in Eq. (4).

Proposition 1. Let $E(\lambda, \ell, \eta)$ be a bound for $\mathcal{R}\left[f_{D}^{\lambda}\right]$, that is, with probability at least $1-\eta$

$$
\mathcal{R}\left[f_{D}^{\lambda}\right] \leq E(\lambda, \ell, \eta) .
$$

Assume that $\lim _{\ell \rightarrow \infty} E(\lambda, \ell, \eta)=A(\lambda)$ for all $\eta$. Then,

$$
\mathcal{R}\left[f^{\lambda}\right] \leq A(\lambda) .
$$

Proof. Let $\epsilon>0$ and $\eta<\frac{1}{2}$. Since $\lim _{\ell \rightarrow \infty} S(\lambda, \ell, \eta)=0$, there is $\ell_{1}$ such that

$$
S(\lambda, \ell, \eta) \leq \epsilon \quad \forall \ell \geq \ell_{1}
$$

and, by definition of $A(\lambda)$, there is $\ell_{2}$ such that

$$
|E(\lambda, \ell, \eta)-A(\lambda)| \leq \epsilon \quad \forall \ell \geq \ell_{2}
$$

Let $\ell_{3}=\max \left\{\ell_{1}, \ell_{2}\right\}$. By Theorem 1 , with probability at least $1-\eta$,

$$
\left|\mathcal{R}\left[f_{D}^{\lambda}\right]-\mathcal{R}\left[f^{\lambda}\right]\right| \leq S\left(\lambda, \ell_{3}, \eta\right) \leq \epsilon
$$

where we used Eq. (30). By definition one has that, with probability at least $1-\eta$,

$$
\mathcal{R}\left[f_{D}^{\lambda}\right] \leq E\left(\lambda, \ell_{3}, \eta\right) \leq A(\lambda)+\epsilon,
$$

where we used Eq. (31).

It follows from Eqs. (32) and (33) that, with probability at least $1-2 \eta>0$,

$$
\begin{aligned}
A(\lambda) & \geq \mathcal{R}\left[f_{D}^{\lambda}\right]-\epsilon \\
& \geq \mathcal{R}\left[f^{\lambda}\right]-2 \epsilon .
\end{aligned}
$$

Since $\epsilon$ is arbitrary, one has the thesis.

According to the discussion in Section 2, given $\eta$ and $\ell$, we estimate the optimal parameter $\lambda_{0}(\ell, \eta)$ as the one that minimizes the bound

$$
E(\lambda, \ell, \eta)=A(\lambda)+S(\lambda, \ell, \eta)
$$

The following proposition shows that $\lambda_{0}$ is finite, at least if $\ell$ is large enough.

Proposition 2. Assume $P f_{0} \neq 0$ and let $0<\eta<1$. There is $\bar{\ell} \in \mathbb{N}$ such that $\lambda_{0}(\ell, \eta)$ is finite for all $\ell \geq \bar{\ell}$.

In particular, if $\lim _{\lambda \rightarrow \infty} A(\lambda)=+\infty$, then $\lambda_{0}(\ell, \eta)$ is finite for every $\ell$.

Proof. We will prove the finiteness of $\lambda_{0}$ applying the Weierstrass theorem to the continuous function $E(\lambda, \ell, \eta)$.

Clearly, $\lim _{\lambda \rightarrow 0} E(\lambda, \ell, \eta)=+\infty$. Moreover, letting $M=\lim _{\lambda \rightarrow \infty} A(\lambda)$, which always exists by the assumed continuity at $\lambda=+\infty$, one has that

$$
\lim _{\lambda \rightarrow \infty} E(\lambda, \ell, \eta)=M
$$

We now prove that, if $\ell$ is large enough, there is $\bar{\lambda}>0$ such that $E(\bar{\lambda}, \ell, \eta)<M$. In fact, since $A(\lambda) \geq \mathcal{R}\left[f^{\lambda}\right]$ and $\lim _{\lambda \rightarrow+\infty} \mathcal{R}\left[f^{\lambda}\right]=\left\|P f_{0}\right\|_{\nu}>0$, then $M>0$. Moreover, since $\lim _{\lambda \rightarrow 0} A(\lambda)=0$, there is $\bar{\lambda}>0$ such that $A(\bar{\lambda})<\frac{M}{2}$. Finally observing that $\lim _{\ell \rightarrow \infty} S(\bar{\lambda}, \ell, \eta)=0$, we conclude that there exists $\bar{\ell} \in \mathbb{N}$ such that

$$
S(\bar{\lambda}, \bar{\ell}, \eta)<\frac{M}{2} .
$$


It follows that, since $S$ is a decreasing function of $\ell$, for all $\ell \geq \bar{\ell}$,

$$
E(\bar{\lambda}, \ell, \eta) \leq E(\bar{\lambda}, \bar{\ell}, \eta)<M
$$

Hence, by means of Weierstrass theorem $E(\lambda, \ell, \eta)$ attains its minimum. Moreover, $\min E \leq E(\bar{\lambda}, \ell, \eta)<M$ so that all the minimizers are finite.

Assume now that $\lim _{\lambda \rightarrow \infty} A(\lambda)=+\infty$, then $M=+\infty$ and, clearly, min $E<$ $+\infty$, so that the minimizers are finite for all $\ell$.

Remark 1. The assumption that $P f_{0} \neq 0$ is natural. If $P f_{0}=0$, the problem of model selection is trivial since $\mathcal{H}$ is too poor to give a reasonable approximation of $f_{0}$ still with infinite data.

In order to actually use the estimate $\lambda_{0}$, we have to explicitly compute the minimizer of the bound $E$. Hence, the function $E$ has to be computable. Though the sample error $S(\lambda, \ell, \eta)$ is a simple function of the parameters $\lambda, \ell, \eta, \kappa$ and $\delta$, the approximation error $\mathcal{R}\left[f^{\lambda}\right]$ is not directly computable and we need a suitable bound.

We do not discuss the problem of the estimate of the approximation error since there is a large literature on the topic, see [15], [19] and references therein. We only notice that a common features of these bounds is that one has to make some assumptions on the probability distribution $\rho$, that is, on the regression function $f_{0}$. Clearly, with these hypotheses we lose in generality. However if we want to solve the bias-variance problem in this framework, it seems to be an unavoidable step (compare with [6], [7], [15]).

Using an estimate on the approximation error $A(\lambda)$ given in Theorem 3, Chapter II of [7], one easily obtains the following result ${ }^{1}$.

Corollary 1. Let $r \in(0,1]$ and $C_{r}>0$ such that $\left\|L_{\nu}^{-r} P f_{0}\right\|_{\nu} \leq C_{r}$, where $f_{0}$ is given by Eq. (9), $L_{\nu}$ and $P$ by Eq. (14), then

$$
\mathcal{R}\left[f_{D}^{\lambda}\right] \leq \lambda^{r} C_{r}+S(\lambda, \ell, \eta)=: E_{r}(\lambda, \ell, \eta)
$$

with probability at least $1-\eta$.

In particular, for all $\ell$ and $\eta$, the bound $E_{r}$ gives rise to a finite estimate $\lambda_{0}^{r}(\ell, \eta)$ of the optimal parameter, which is the unique solution of the following equation

$$
r C_{r} \lambda^{r+1}=\frac{\delta \kappa^{2}}{\sqrt{\ell}}\left(1+\frac{3 \kappa}{2 \sqrt{\lambda}}\right)\left(1+\sqrt{2 \log \frac{2}{\eta}}\right) .
$$

To compare our results with the bounds obtained in the literature we assume that $f_{0}$ belongs to the hypothesis space so we can choose $r=\frac{1}{2}$ in the above corollary. Since $I_{\mathcal{H}}=I\left[f_{0}\right], I[f]=\mathcal{R}[f]^{2}+I\left[f_{0}\right]$ so that

$$
I\left[f_{D}^{\lambda}\right]=I\left[f_{0}\right]+O(\lambda)+O\left(\frac{1}{\ell \lambda^{3}}\right)
$$

with probability greater than $1-\eta$. The best rate convergence is obtained by choosing $\lambda_{\ell}=\frac{1}{\sqrt[4]{\ell}}$ so that

$$
I\left[f_{D}^{\lambda}\right]=I\left[f_{0}\right]+O\left(\frac{1}{\sqrt[4]{\ell}}\right)
$$

\footnotetext{
${ }^{1}$ In Appendix A we provide a direct proof of such an estimate.
} 
The rate is comparable with the rate we can deduce from the bounds of [4] where, however, the dependence of $\lambda$ from $\ell$ is not considered ${ }^{2}$. In [23] a bound of the order $0\left(\frac{1}{\sqrt{n}}\right)$ is obtained using a leave-one-out technique, but with a worse confidence level ${ }^{3}$.

Finally, we notice that our model selection rule, based on a-priori assumption on the target function, is only of theoretical use since the condition that the approximation error is of the order $O\left(\lambda^{r}\right)$ is unstable with respect to the choice of $f_{0}$ (if the kernel is infinite dimensional, as the Gaussian kernel), see [19].

4.1. Asymptotic properties and consistency. The aim of the present subsection is stating some asymptotic properties, for increasing number of examples $\ell$, of the regularized least-squares algorithm provided with the parameter choice described at the beginning of this Section. In particular we consider properties of the selected parameter $\lambda_{0}=\lambda_{0}(\ell, \eta)$ with respect to the notion of consistency already introduced by Definition 1 of Section 2. For clarity we restate here that definition in terms of the modified expected risk $\mathcal{R}\left[f_{D}^{\lambda}\right]$ defined in Section 3.

Definition 2. The one parameter family of estimators $f_{D}^{\lambda}$ provided with a model selection rule $\lambda_{0}(\ell)$ is said to be (weakly universally) consistent if, for every $\epsilon>0$, it holds

$$
\lim _{\ell \rightarrow \infty} \sup _{\rho} \operatorname{Prob}\left\{D \in Z^{\ell} \mid \mathcal{R}\left[f_{D}^{\lambda_{0}(\ell)}\right]>\epsilon\right\}=0
$$

where the sup is over the set of all probability measures on $X \times Y$.

From a general point of view consistency can be considered as a property of the algorithm according to which for large data amount the algorithm provides the best possible estimator.

In order to apply this definition to our selection rule we need to specify the explicit dependence of the confidence $\eta$ on the number of examples $\ell$, i.e. to transform the two-parameter family of real positive numbers $\lambda_{0}(\ell, \eta)$ to the one-parameter family $\lambda_{0}(\ell)=\lambda_{0}(\ell, \bar{\eta}(\ell))$ corresponding to a specific choice $\bar{\eta}(\ell)$ of the confidence level. We assume the following power law behavior:

$$
\bar{\eta}(\ell)=\ell^{-p} p>0 .
$$

The main result of this section is contained in the following theorem where we prove that the regularized least-squares algorithm algorithm provided by our model selection rule is consistent.

Theorem 2. Given $\lambda_{0}(\ell)=\lambda_{0}(\ell, \bar{\eta}(\ell))$ where $\bar{\eta}(\ell)$ as in Eq. (34), then the following three properties hold:

(1) if $\ell^{\prime}>\ell>2$, then $\lambda_{0}\left(\ell^{\prime}\right) \leq \lambda_{0}(\ell)$;

(2) $\lim _{\ell \rightarrow \infty} \lambda_{0}(\ell)=0$;

(3) the sequence $\left(\lambda_{0}(\ell)\right)_{\ell=1}^{\infty}$ provides consistency.

Proof. First of all let us notice that the dependence of $E(\lambda, \ell, \eta)$ on $\ell$ and $\eta$ can be factorized as follows

$$
E(\lambda, \ell, \eta)=A(\lambda)+C(\ell, \eta) s(\lambda)
$$

\footnotetext{
${ }^{2}$ see Theorem 12 and 22 of [4], in particular the proof of the latter theorem gives an upper bound of the sample error of the order $O\left(\frac{1}{\sqrt{\ell} \lambda^{\frac{3}{2}}}\right)$.

3 see discussions at the end of Sections 4 and 5 of [23]
} 
where the sample error term $S(\lambda, \ell, \eta)$ in Eq. (17) has been split by means of the functions $C(\ell, \eta)$ and $s(\lambda)$ defined by

$$
\begin{gathered}
C(\ell, \eta)=\frac{\delta}{\sqrt{\ell}}\left(1+\sqrt{2 \log \frac{2}{\eta}}\right), \\
s(\lambda)=\frac{\kappa^{2}}{\lambda}\left(1+\frac{\kappa}{\sqrt{\lambda}}\right) .
\end{gathered}
$$

In order to prove the first part of the Theorem we show that, if $\ell^{\prime}>\ell>2$, then

$$
E\left(\lambda, \bar{\eta}\left(\ell^{\prime}\right), \ell^{\prime}\right)>E\left(\lambda_{0}(\ell), \bar{\eta}\left(\ell^{\prime}\right), \ell^{\prime}\right) \text { for every } \lambda>\lambda_{0}(\ell),
$$

implying that the minimizer of $E\left(\lambda, \bar{\eta}\left(\ell^{\prime}\right), \ell^{\prime}\right), \lambda_{0}\left(\ell^{\prime}\right)$, is not greater than $\lambda_{0}(\ell)$, as claimed. Inequality (38) can be proved considering the identity

$$
E\left(\lambda, \bar{\eta}\left(\ell^{\prime}\right), \ell^{\prime}\right)=E(\lambda, \bar{\eta}(\ell), \ell)-\left(C(\bar{\eta}(\ell), \ell)-C\left(\bar{\eta}\left(\ell^{\prime}\right), \ell^{\prime}\right)\right) s(\lambda) .
$$

First of all, we observe that, due to the power law behavior of $\bar{\eta}(\ell)$, the function $C(\bar{\eta}(\ell), \ell)=\frac{\delta}{\sqrt{\ell}}\left(1+\sqrt{2 \log \left(2 \ell^{p}\right)}\right)$ is strictly decreasing for $\ell>2$, so that under the conditions on $\ell^{\prime}$ and $\ell$ in the text of the Theorem, the difference $C(\bar{\eta}(\ell), \ell)-$ $C\left(\bar{\eta}\left(\ell^{\prime}\right), \ell^{\prime}\right)$ is positive. Moreover, since $s(\lambda)$ is a strictly decreasing function and due to the definition of $\lambda_{0}(\ell)$ as the maximum of the minimizers of $E(\lambda, \bar{\eta}(\ell), \ell)$ (Eq. (6)), we can bound the two terms in the previous equality as follows

$$
E\left(\lambda, \bar{\eta}\left(\ell^{\prime}\right), \ell^{\prime}\right)>E\left(\lambda_{0}(\ell), \bar{\eta}(\ell), \ell\right)-\left(C(\bar{\eta}(\ell), \ell)-C\left(\bar{\eta}\left(\ell^{\prime}\right), \ell^{\prime}\right)\right) s\left(\lambda_{0}(\ell)\right)
$$

for every $\lambda>\lambda_{0}(\ell)$. Using again Eq. (39), the previous inequality reduces to Eq. (38) as required.

We now prove the remaining two parts of the Theorem. At this purpose we produce a sequence $(\bar{\lambda}(\ell))_{\ell=1}^{\infty}$ such that

$$
\lim _{\ell \rightarrow+\infty} E(\bar{\lambda}(\ell), \bar{\eta}(\ell), \ell)=0, .
$$

Since, by the definition of $\lambda_{0}(\ell), E\left(\lambda_{0}(\ell), \bar{\eta}\left(\ell^{\prime}\right), \ell^{\prime}\right)$ is not greater than $E\left(\bar{\lambda}(\ell), \bar{\eta}\left(\ell^{\prime}\right), \ell^{\prime}\right)$, a fortiori the following limit holds

$$
\lim _{\ell \rightarrow+\infty} E\left(\lambda_{0}(\ell), \bar{\eta}(\ell), \ell\right)=0 .
$$

Moreover, since $A(\lambda)$ is bounded from above by $E(\lambda, \ell, \eta)$, also $A(\lambda)$ vanishes for increasing $\ell$. From the last fact the second part of the theorem follows recalling that $A(\lambda)$ is an increasing function of $\lambda$ and $A(0)=0$ (see beginning of this Section).

Equation (41) also ensures consistency of the sequence $\left(\lambda_{0}(\ell)\right)_{\ell=1}^{\infty}$ since, by our definition of the probabilistic bound $E(\lambda, \ell, \eta)$, we can write

$$
\operatorname{Prob}\left\{D \in Z^{\ell} \mid \mathcal{R}\left[f_{D}^{\lambda_{0}(\ell)}\right]>E\left(\lambda_{0}(\ell), \bar{\eta}(\ell), \ell\right)\right\} \leq \bar{\eta}(\ell)
$$

and moreover $\bar{\eta}(\ell)$ goes to zero as $\ell$ goes to infinity. It remains to show a sequence verifying Eq. (40). Let us choose the following $\bar{\lambda}(\ell)$

$$
\bar{\lambda}(\ell)=\ell^{-q} \text { with } 0<q<\frac{1}{3} .
$$

First, since $\bar{\lambda}(\ell)$ vanishes as $\ell$ increases, then the approximation term $A(\bar{\lambda}(\ell))$ goes to zero. Moreover, recalling the representation in Eq. (35), we have to show that

$$
\lim _{\ell \rightarrow+\infty} C(\bar{\eta}(\ell), \ell) s(\bar{\lambda}(\ell))=0
$$


this fact can be directly verified by substitution of the expressions of $\bar{\lambda}$ and $\bar{\eta}$ into the functions $s$ and $C$.

The previous result reduces to the approximation error bound considered in Corollary 1 . In this case the computable bound $E_{r}$ given in Corollary 1 allows us to obtain the explicit asymptotic form for the selected $\lambda_{0}$.

Theorem 3. Given the sequence $\left(\lambda_{0}^{r}(\ell)\right)_{\ell=1}^{\infty}$ such that $\lambda_{0}^{r}(\ell)$ minimizes the bound $E_{r}(\lambda, \bar{\eta}(\ell), \ell)$ of Corollary 1 then we have that

(1) for all $\ell>0, \lambda_{0}^{r}(\ell)$ is finite;

(2) $\lambda_{0}^{r}(\ell)=\ell^{-\frac{1}{3+2 r}}\left(\frac{3 \kappa^{3} \delta}{2 r C_{r}}\left(1+\sqrt{2 \log \left(2 \ell^{p}\right)}\right)\right)^{\frac{2}{3+2 r}}+O\left(\ell^{-\frac{3}{2(3+2 r)}}\right)$.

Proof. As in the proof of Theorem 2 we introduce the functions $C$ and $s$, defined in Eqs. (36)- (37), to factorize in $E_{r}$ the dependence on $\ell$ and $\eta$, that is we write

$$
E_{r}(\lambda, \ell, \eta)=\lambda^{r} C_{r}+C(\ell, \eta) s(\lambda) .
$$

Then, the first part of the Theorem follows immediately from Proposition 2 as a consequence of the divergence of $E_{r}$ for increasing $\lambda$.

The second part can be proved exploiting the differentiability of $E_{r}$ with respect to $\lambda$. Since $\lambda_{0}^{r}(\ell)$ is a minimizer of $E_{r}$, it must be a zero of its derivative. By explicit differentiation we obtain that $\lambda_{0}^{r}(\ell)$ is a solution of the following algebraic equation

$$
2 r C_{r} \kappa^{-2} \lambda^{\frac{3+2 r}{2}}=C(\bar{\eta}(\ell), \ell)\left(2 \lambda^{\frac{1}{2}}+3 \kappa\right) .
$$

It is convenient reformulating the last equation in terms of the auxiliary variables $x(\ell)$ and $y(\ell)$ defined by

$$
\begin{gathered}
x(\ell)=\lambda C(\bar{\eta}(\ell), \ell)^{-\frac{2}{3+2 r}}, \\
y(\ell)=C(\bar{\eta}(\ell), \ell)^{\frac{1}{3+2 r}} .
\end{gathered}
$$

Using this notation Eq. (42) becomes

$$
r C_{r} \kappa^{-2} x(\ell)^{1+r}-\frac{3}{2} \kappa x(\ell)^{-\frac{1}{2}}=y(\ell) .
$$

The function of the unknown $x(\ell)$ at the r.h.s. of Eq. (43) has positive derivative, moreover it assumes arbitrary real values as its argument range the positive real numbers. This fact proves that, for a given $\ell$ there exists a unique solution $x(\ell)$. This also implies that $E_{r}$ has a unique finite minimizer.

Since by definition $y(\ell)$ is $O\left(\ell^{-\frac{1}{2(3+2 r)}}\right)$, we deduce that for increasing $\ell, x(\ell)$ goes to the zero of the r.h.s of Eq. (43), in fact to the value

$$
x_{0}=\left(\frac{3 \kappa^{3}}{2 r C_{r}}\right)^{\frac{2}{3+2 r}} .
$$

Finally, due to the regular behavior of the function in Eq. (43) in $x_{0}$, we can write

$$
x(\ell)=x_{0}+O\left(\ell^{-\frac{1}{2(3+2 r)}}\right),
$$

that provides the claimed result. 


\section{Conclusions}

In this paper we focus on a functional analytical approach to learning theory, in the same spirit of [7]. Unlike other studies we do not examine the deviation of the empirical error from the expected error, but we analyze directly the expected risk of the solution. As a consequence the splitting of the expected error in an estimation error term and an approximation error follows naturally giving rise to a bias-variance problem.

In this paper we show a possible way to solve this problem by proposing a model selection criterion that relies on the stability properties of the regularized leastsquares algorithm and does not make direct use of any complexity measures. In particular our estimates depend only on a boundedness assumption on the output space and the kernel.

Our analysis uses extensively the special properties of the square loss function, henceforth it would be interesting to extend our approach to other loss functions. We think that our results may be improved by taking into account more information about the structure of the hypothesis space.

Acknowledgment 1. We thank for many useful discussions and suggestions Michele Piana, Alessandro Verri and the referees. L. Rosasco is supported by an INFM fellowship. A. Caponnetto is supported by a PRIN fellowship within the project "Inverse problems in medical imaging", n. 2002013422. This research has been partially funded by the INFM Project MAIA, the FIRB Project ASTA, and by the EU Project KerMIT.

\section{Appendix A. Bounding the APPROXimation ERror}

In this appendix we report an elementary proof of the following result from approximation theory.

Theorem 4. Assume that there is $r \in(0,1]$ such that $P f_{0}$ is in the domain of $L_{\nu}^{-r}$ and let $C_{r}$ be a constant such that $\left\|L_{\nu}^{-r} P f_{0}\right\|_{\nu} \leq C_{r}$. Then,

$$
\mathcal{R}\left[f^{\lambda}\right] \leq \lambda^{r} C_{r}
$$

Proof. Starting from Eq. (21) in section 3.3 and the definition of $C_{r}$ we just have to show that

$$
\left\|f^{\lambda}-P f_{0}\right\|_{\nu} \leq \lambda^{r}\left\|L_{\nu}^{-r} P f_{0}\right\|_{\nu}
$$

Due to the fact that $K$ is a Mercer kernel, $L_{\nu}$ is a compact positive operator and, by spectral decomposition of $L_{\nu}$, there is a sequence $\left(\phi_{n}\right)_{n=1}^{N}$ in $L^{2}(X, \nu)$ (possibly $N=+\infty)$ such that

$$
\begin{gathered}
\left\langle\phi_{n}, \phi_{m}\right\rangle_{\nu}=\delta_{n m} \\
L_{\nu} f=\sum_{n=1}^{N} \sigma_{n}^{2}\left\langle f, \phi_{n}\right\rangle_{\nu} \phi_{n},
\end{gathered}
$$

where $\sigma_{n} \geq \sigma_{n+1}>0$. In particular, $\left(\phi_{n}\right)_{i=1}^{N}$ is a orthonormal basis of the range of $P$.

Moreover, since the function $g(x)=x^{r}$ is a concave function on $(0,+\infty)$ and $g^{\prime}(1)=r$, then

$$
x^{r} \leq r(x-1)+1 \leq x+1 .
$$


Recalling that

$$
\begin{aligned}
& f^{\lambda}=\left(L_{\nu}+\lambda\right)^{-1} L_{\nu} f_{0} \\
\left\|f^{\lambda}-P f_{0}\right\|_{\nu}^{2} & =\sum_{n=1}^{N}\left\langle\left(\left(L_{\nu}+\lambda\right)^{-1} L_{\nu}-I\right) P f_{0}, \phi_{n}\right\rangle_{\nu}^{2} \\
& =\sum_{n=1}^{N}\left(\frac{\sigma_{n}^{2}}{\sigma_{n}^{2}+\lambda}-1\right)^{2}\left\langle P f_{0}, \phi_{n}\right\rangle_{\nu}^{2} \\
\left(x_{n}=\frac{\sigma_{n}^{2}}{\lambda}\right) & =\sum_{n=1}^{N}\left(\frac{1}{x_{n}+1}\right)^{2}\left\langle P f_{0}, \phi_{n}\right\rangle_{\nu}^{2} \\
(\mathrm{Eq} .(44)) \leq & \sum_{n=1}^{N}\left(\frac{1}{x_{n}^{r}}\right)^{2}\left\langle P f_{0}, \phi_{n}\right\rangle_{\nu}^{2} \\
= & \lambda^{2 r} \sum_{n=1}^{N}\left(\frac{1}{\left(\sigma_{n}^{2}\right)^{r}}\right)^{2}\left\langle P f_{0}, \phi_{n}\right\rangle_{\nu}^{2} \\
= & \lambda^{2 r}\left\|L_{\nu}^{-r} P f_{0}\right\|_{\nu}^{2} .
\end{aligned}
$$

\section{REFERENCES}

[1] N. Alon, S. Ben-David, N. Cesa-Bianchi, and D. Haussler, Scale-sensitive dimensions, uniform convergence, and learnability, J. ACM 44 (1997), 615-631.

[2] N. Aronszajn, Theory of reproducing kernels, Trans. Amer. Math. Soc. 68 (1950), 337-404.

[3] P. Bartlett, S. Boucheron, and G. Lugosi, Model selection and error estimation, Machine Learning 48 (2002), 85-113.

[4] O. Bousquet and A. Elisseeff, Stability and generalization, J. Mach. Learn. Res. 2 (2002), $499-526$.

[5] N. Cristianini and J. Shawe Taylor, An Introduction to Support Vector Machines, Cambridge University Press, Cambridge, UK, 2000.

[6] F. Cucker and S. Smale, Best choices for regularization parameters in learning theory: on the bias-variance problem, Found. Comput. Math. 2 (2002), 413-428.

[7] F. Cucker and S. Smale, On the mathematical foundations of learning, Bull. Amer. Math. Soc. (N.S.) 39 (2002), 1-49 (electronic).

[8] L. Devroye, L. Györfi, and G. Lugosi, A probabilistic theory of pattern recognition, SpringerVerlag, New York, 1996.

[9] R. Dudley, Real analysis and probability, Cambridge University Press, Cambridge, 2002.

[10] T. Evgeniou, M. Pontil, and T. Poggio, Regularization networks and support vector machines, Adv. Comput. Math. 13 (2000), 1-50.

[11] F. Girosi, M. Jones, and T. Poggio, Regularization theory and neural networks architectures, Neural Computation 7 (1995), 219-269.

[12] T. Hastie, R. Tibshirani, and J. Friedman, The elements of statistical learning, SpringerVerlag, New York, 2001.

[13] C. McDiarmid, On the method of bounded differences, In Surveys in combinatorics, 1989 (Norwich, 1989), Cambridge Univ. Press, Cambridge, 1989, pp. 148-188.

[14] S. Mendelson, A few notes on statistical learning theory, In S. Mendelson and A. Smola, editors, Advanced Lectures in Machine Learning, Springer-Verlag, 2003, pp. 1-40.

[15] P. Niyogi and F. Girosi, Generalization bounds for function approximation from scattered noisy data, Adv. Comput. Math. 10 (1999), 51-80.

[16] T. Poggio and F. Girosi, Networks for approximation and learning, In Proceedings of the IEEE, volume 78, 1990, pp. 1481-1497.

[17] T. Poggio, R. Rifkin, S. Mukherjee, and P. Niyogi, General conditions for predictivity in learning theory, Nature 428 (2004), 419-422. 
[18] L. Rosasco, E. De Vito, A. Caponnetto, M. Piana, and A. Verri, Are loss functions all the same?, Neural Computation 16 (2004), 1063-1076.

[19] S. Smale and D.-X. Zhou, Estimating the approximation error in learning theory, Anal. Appl. (Singap.) 1 (2003), 17-41.

[20] I. Steinwart, Consistency of support vector machines and other regularized kernel machines, Technical Report 02-03, University of Jena, Department of Mathematics and Computer Science, 2002.

[21] V. Vapnik, Statistical learning theory, John Wiley \& Sons Inc., New York, 1998.

[22] G. Wahba, Spline models for observational data, Society for Industrial and Applied Mathematics (SIAM), Philadelphia, PA, 1990.

[23] T. Zhang, Leave-one-out bounds for kenrel methods, Neural Computation 15 (2003), 13971437.

[24] D.-X. Zhou, The covering number in learning theory, J. Complexity 18 (2002), 739-767.

Ernesto De Vito, Dipartimento di Matematica, Università di Modena, Via Campi 213/B, 41100 Modena, Italy and I.N.F.N., Sezione di Genova, Via Dodecaneso 33, 16146 Genova, Italy,

E-mail address: devito@unimo.it

Andrea Caponnetto, D.I.S.I., Università di Genova, Via Dodecaneso 35, 16146 Genova, Italy, and I.N.F.M., Sezione di Genova, Via Dodecaneso 33, 16146 Genova, Italy

E-mail address: caponnetto@disi.unige.it

Lorenzo Rosasco, D.I.S.I., Università di Genova, Via Dodecaneso 35, 16146 Genova, Italy, and I.N.F.M., Sezione di Genova, Via Dodecaneso 33, 16146 Genova, Italy

E-mail address: rosasco@disi.unige.it 